\title{
A PRODUC̣ÃO CIENTÍFICA E A ENFERMAGEM OCUPACIONAL
}

Maria do Socorro Batist a de Souza *

\begin{abstract}
RESUMO -Estuda a produção científica na área de Enfermagem Ocupacional, no período de 1941 a 1986. Informa sobre as mudanças que ocorreram ao longo da história da Enfermagem. Seleciona material bibliográfico para pesquisa e realiza levantamento da produção científica sobre Enfermagem Ocupacional. Constatada a escassez da produção científica e as dificuldades para difusão e acesso aos conhecimentos na área da Enfermagem Ocupacional.
\end{abstract}

ABSTRACT - It studies the scientifical production in the area of Occupational Nursing during the period 1941 - 1986. It informs about changes that have occurred along the history of Nursing. It selects bibliographic material for research and carries out a survey of scientifical production on Ocupational Nursing. It shows the for diffusion and approach of knowledge in the area of Occupational Nursing.

\section{INTRODUC̣ÃO}

O desenvolvimento da ciência e da tecnologia, bem como as crescentes modificações de toda uma estrutura social vem causando uma verdadeira explosão de conhecimentos, além de vastas e profundas transformaçōes em todos os campos do conhecimento humano.

As mudanças que ocorreram ao longo da história da Enfermagem e em especial da Enfermagem Ocupacional fazem com que o enfermeiro compartilhe com outros prof issionais da responsabilidade dos rumos dos programas educacionais e assistenciais, a partir do desafio que solicita novas abordagens.

Como conseqüência disso, acredita-se na necessidade de busca constante de atualização de conhecimentos para que se possa estabelecer a sistematização dos mesmos, além de efetivar uma rotina de trabalho e assim acompanhar a evolução técnico-científicohumanística dessa especialidade.

Considerou-se o material disponivel de informaçōes na Enfermagem Ocupacional, com grande representatividade para fornecer uma perfeita compreensão da

\footnotetext{
* Mestre em Enfermagem pela UFRJ, Professora Adjunta II do Departamento de Enfermagem do Centro de Ciência da Saúde da Uni versidade Federal do Pará. COREN-PA 1968.
}

realidade brasileira, e construir uma memória científica a partir do levantamento de documentos publicados, procurando colocar o estudioso em contato com tudo aquilo que já foi escrito a fim de evitar duplic a(cão de pesquisas sobre o mesmo tema.

Iessa forma, poder-se-á direcionar estudos futuros e permitir maior instrumentalização à pesquisa, para que a mesma se constitua num núcleo de estímulos e retroalimenta(ção. Diante disso, deve-se considerar as ('ontribuiçoes anteriores, refletir sobre elas e extrair conclusoès út eis e necessárias, as quais aument arão a produção da nossa literatura especializada.

Para analisar a produção científica da Enfermagem ()cupacional, é necessário que levantemos os seguintes pressupostos:

a. Estão os enfermeiros elaborando trabalhos científicos, na área de Enfermagem ()cupacional'?

b. A elaboração teórica da Enfermagem () ('upacional, sofre a influência da pobreza bibliográfic “a”.

c. A não divulgação de pesquisas realizadas, impede ou prejudica a continuidade de estudos ne'ssa área? Para isso, é objetivo do presente estudo:

a. Identificar e consultar guias bibliográficos (índicess e abstracts) sobre enfermagem, notadamente a Enfermagem ()cupacional;

b. Realizar levantamento bibliográfico sobre Enfermagem Ocupacional, no seu aspecto exaustivo;

c. Identificar as publicaçōes periódicaas onde a litera- 
tura produzida sobre Enfermagem ( )(upacional é public'adla;

d. ()ferecer subsídio aos enfermeiros que bus('am aponoio da literatura para possíveis estudos da Enfermagem ()cupacional.

\section{REVISÃO DE LITERATURA}

A importâncià do acessso à difusào de novos conhe(imentos levaram LANCASTER (1975), A(iUIARI (1981) WRI(iIIT et alii (1981) e ANGERAMI \& ALMEII)A (1982) a reconhecer no "processo de acessibilidade e velocidacle da informação, ser o periódico científico o material bibliográfico mais importante no acervo de uma biblioteca espeecializada, (ladla a sua ('ontribuiçào efetiva, tanto para o ensino, (omo para a pesquisa". Referem-se, ainda, ser o mesmo "o veículo de ('omuni('a(̧ão eficciente, entre os profissionais das diversas áreas do conhecimento, permitindo que o fluxo de idéias e informaçoes de grande numero de pesquisas se faça de maneira rápicla, freqüente e regular, estimulando novos trabalhos sobre os quais se alcançam os avanços (científicos". Afirmam os autores que o volume de títulos editados provocas sérias dificuldades para os estudiosos que precisam dispender tempo ("ala vez maior para selecionar, dentre a massa de artigos publicados, os novos conhecimentos e idéias de que necessit am para se manterem at ualizados.

PAIM (1979) ressalta ser a "produção científica na Enfermagem, incipiente"'. (O assunto é enfatizado por RODRIGUES (1981) WRIGHT et alii (1981) ARGERAMI \& ALMEIDA (1982) que mencionam ser insuficiente 0 acesso à literatura que serve de base às fundamentações e à dicussão de problemas de pesquisa em Enfermagem. Abordam a pouca atenção dada aos resultados já efetuados que podem representar uma limitação ao desenvolvimento da prática da pesquisa em Enfermagem, o que se tornou motivo de estudos também por CIANCIARULLO (1979) ALMEIDA (1985) NÓBREGA (1985) CASTR() et alii (1985) os quais "responsabilizaram a não divulgação de pesquisas realizadas, como impedimento de estudos sobre questões que não tenham sido bem tratadas'.

CARVALHO \& BARRETO (198()) realizaram estudos referentes ao conhecimento de indicadores de comunicação científica. Lembram que "os pesquisadores usuários que trabalham nas diversas áreas do conhecimento, são também geradores de informações, pois sempre descrevem os resultados de seus trabalhos em algum tipo de documento"'. Referem que neste caso os pesquisadores desempenham papel do autor, no ato da transferência da informação. Afirmam os autores que para causar impacto na comunidade, o documento necessita ser reproduzido e distribuído por órgão editor. Reforçados por FERREIRO \& MENDÉZ (1980) enfatizam que o aumento da literatura científica representa o caráter exponencial que só recentente é objeto de estudo em profundidade. Lembram que é a partir de dados bibliográficos que se facilita a pesquisa científica.

FURTADO (1981) refere que "no sistema científico a publicação é o principal indicador, por excelência profissional e o meio possível de alcançar níveis mais altos na hierarquia". Diz ele: "na produção e publicação de artigos, ou conhecimentos do assunto, viver o problema é motivar-se"'; posteriormente deve-se selecionar informações relevantes e permanentes. Alerta para produção da informação marginal, constituída por resultados triviais, imcompetência de técnicas experimentais ou dados permanentes, tecnológicos e comerciais, o que é ressaltado, também, por ANGERAMI \& ALMEIDA (198:).

SANTOS (1981) refere que a atividade científica é af etada se a comunicação de dados ou informações não se processar adequadamente. Para o autor "Se não há estímulo institucional, o intercâmbio de idéias é dificultado, bem como a divisão de novos conhecimentos e acesso aos resultados produzidos"'. Desta forma, os pesquisadores necessitam seguir de perto os progressos realizados em sua área de especialização.

SANTOS (1981) cita BARBER (1962) e GIANOTTI (1975), que enfatizam a necessidade dos estudiosos serem reconhecidos e terem contatos diretos com seus colegas ou pares do País ou exterior, reforçado por MOREL \& MOREL (1977), para que possam integrar-se nas áreas afins. MERTA (1972) acrescenta, ainda, que a troca de informações ou seu intercâmbio é fundamental para o progresso da ciência. Lembra a autora, a necessidade de instrumentos formais de comunicação que lhes permitam acompanhar trabalhos desenvolvidos por seus pares. Refere ainda PRICE (1986), que “a publicação não é apenas o produto final do trabalho do cientista, mas também a garantia de sua propriedade para o trabalho científico que produziu e o meio pelo qual obterá reconhecimento". Diz que a maior parte dos resultados das pesquisas são publicadas em revistas especializadas.

WRIGHT et alii (1981) referem haver "necessidade de estudos alternativos, para compor um sistema de publicações e divulgação apoiado na infra estrutura da ciência e da tecnologia que permita a documentação, circuçação e difusão da Enfermagem como profissão científica". Afirmam que "a divulgação do conhecimento à comunidade científica é essencial para que seja submetido à crític ‘'. Consideram que o desenvolvimento progressivo de uma ciência consiste no aumento do volume e finalidade de sua produção, para medir o avanço nessa área. Lembram as várias implic‘açoes que a publicação traz e dizem que o "pesquisador deve publicar o seu trabalho quando estiver amadurecido e consistente"

ANGERAMI \& ALMEIDA (1982) citam SALOMON, o qual conceitua Divulgação Científica como: "a comunicação ao público em geral ou particular de conhecimentos extraídos de obras de investigação científica 
com o fim de informar". Enfatizam a preocupação dos cientistas da informação que buscam solução a curto, médio e longo prazo. Mencionam BÜNGE dizendo que o "desenvolvimento científico consiste de aumento do volume e qualidade da produção científica da comunidade". Refere os autores que "os enfermeiros também publicam seus trabalhos em revistas não específicas de enfermagem o que torna de maior amplitude a divulgação de seus estudos e ao mesmo tempo difi("ulta sua recuperação e compilação)" pois sua referen('iação) encontra-se em índices gerais. Afirmam os autores que "não há falta de trabalhos para publicação, há falta de pessoal de apoio para a maior duvulgação da revist a", trabalhos a ademicos como teses, gue são (atalogadas, exposiçoes orais a través de reuniōes científicas, congressos, painéis, mesas redondas, conferên('ias, seminários e outros.

Ressalt am a vantagem da publicação em periódi('os. Relat am que a área da Enfermagem recorre a consult as ao Centro Latino Americano de Informação em ( Giências da Saúde e as bibliotecas que dispoem de índicese e "abstracts", entre estes o Internacional Nursing Index, o ('umulative Index to Nursing, e Allied Ilealt h Litterature Nursing Index, o Cumulative Index to Nursing, e Allied Health Litterature, além da edi(̧ão) do Index Medicus Latino Americano, que vem sen(do publicado desde 1979 e onde estão indexadas as principais publicaçoess periódicas sobre Enfermagem. I)est a('am ('omo revistas brasileiras indexadas: Enfermagem Novas I Dimensões, publicada pela Escola de Enfermagem de São Paulo, a Revita Paulista de Enfermagem e a Revista Brasileira de Enfermagem sendo esta última a mais antiga e tradicional. Referem estudos realizados por bibliotecários da Universidade Federal da Bahia, revelando ser a mesma a mais consultada naquela Instituição. ('onstat aram os autores, em estudos realizados com edit ores de revista brasileiras ser o fator financeiro responsável por atrasos e interrupçōes, além da escolha da composição do Conselho Editorial. Lembram que em matéria de divulgação da enfermagem existem " os ("atálogos de teses editadas pelo Centro de Pesquisas em Enfermagem - CEPEN (órgão de Asssociação Brasileira de Enfermagem - ABEn), Boletins Informativos, Jornais Especializados e Anais de ('ongressos de Enfermagem". Enfatizam que os meios de comunica(ção têm sido muito pouco utilizados pela área de enfermagem, para haver reconhecimento da profissão, a fim de que a população conheça seu trabalho e perceba sua utilidade e o papel social do enfermeiro, no que também são acompanhadas por WRIGHT et alii (1981).

FERREIRA (1982) (aracteriza "a atividade científica, sempre por uma intensa troca de informaçōes entre os pesquisadores os quais se reunem com o propósito de discutir seus problemas e resultados". Afirma que em nosso País pouca importância tem sido dada a qualquer tipo de registro; aqueles referentes às atividades acadêmicas, não constituem exceção. Menciona o autor a necessidade do conhecimento do potencial científico e de estudos, em função dessas informações.

ALMEIDA (1985) recomenda aos enfermeiros a importância de uma base teórica, ao implementar resultados de uma pesquisa. Menciona KING et alii (1981), responsabilizando "a pouca comunicação das informaçóes dos pesquisadores para os enfermeiros "consumidores", dizendo que as pesquisas levam muito tempo para serem comunicadas e quando os resultados destas são informados tendem a responder a uma questão hipotétic(a, assunto também enfatizado por MAIJEIRA (1985). Lembra a autora, que o "meio de comunicação deverá ser capaz de influenciar e enriquecer uma população significante da contribuição que se pretende dar, aos aspectos de mudança da prática de enfermagem". Constata, ainda, em seus estudos, que "os profissionais de enfermagem desconhecem, em grande parte, os resultados das pesquisas sobre sua própria profissão e quando os conhecem, não os utilizam". Refere que os meios de divulgação na profissão não permitem uma boa comunica(ção.

NÓBREGA (1985) destaca que "ninguém deve se considerar dono de um conhecimento científico pois assim estaria negando o cunho social da ciência". Afirma que, quanto mais uma descoberta é divulgada, maior se torna o raio de possibilidade de sua utiliza(̧ão) em suscitar a necessidade de novos estudos para ratificar a sua veracidade e torná-la universal. Men(iona, ainda, que algumas vezes a publicação do trabalho não é sat isfatória e que a curiosidade do pesquisador, porém, servirá de indicador de onde pode ser localizado o seu autor", o qual poderá fornecer melhores informaçōes sobre o assunto.

Elabora o autor um ciclo sistêmáticoo (Fig. 1) para fut uros est udos onde uma melhor orient ação permitirá a identificação da característica cumulativa do conhecimento ('ientífico e a importância da divulgação) dos result ados da investigação.

(ASTR() (1985) afirma que "lendo publicações é que se tornam familiarizados tanto os dados apresentados quanto os seus autores, os quais poderão ser convidados a apresentar seus estudos a comunidade int eressadla".

\section{MATERIAL}

Utilizou-se como material bibliográgico, para levantamento da produção científica na área da enfermagem ocupacional, publicações periódicas, catálogos do (entro de Pesquisas em Enfermagem (CEPEN), programas Anais: de Congresso Nacionais de Prevenção de Acidentes de Trabalho - CoNPAT, Boletim da Associa(ção) Brasileira de Enfermagem - ABEN e Boletins especializados, na área. 


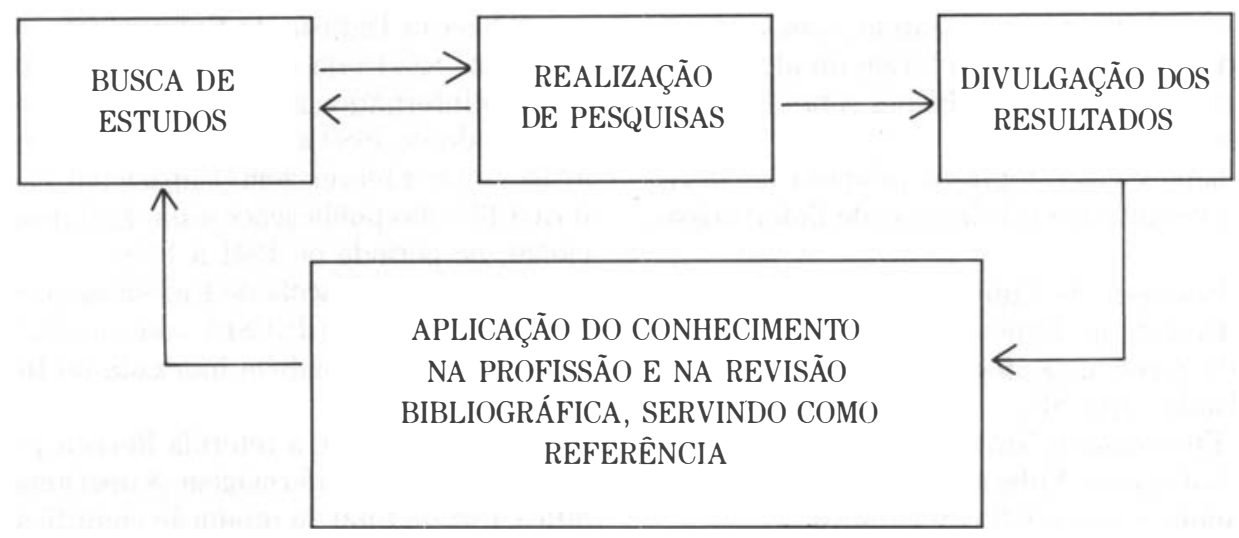

Excluem-se os livros didáticos, por se constituirem fonte secundária de informações.

\section{MÉTOdOS}

4.1. Utilizamos o método de amostragem, selecionandose sete (07) revista nacionais, sendo destas as cinco (05) mais difundidas do saber do enfermeiro e duas (02) da área de Saúde Ocupacional.

4.2. Como complemento, utilizamos a busca direta através do Catálogo do Centro de Pesquisa em Enfermagem (CEPEN), Anais de Congressos Nacionais: de Pre- venção de Acidentes do Trabalho - CONPAT e de Enfermagem, além da pesquisa direta em Boletins especializados, nessa área.

4.3. Adotamos como arranjo para o levantamento bibliográfico efetuado, a ordem cronológica em que foram publicados os trabalhos.

\section{RESULTADOS}

Analisando o material que consultamos, chegamos ao seguinte resultado:

TABELA 1- Trabalhos Publicados sobre Enfermagem Ocupacional, no período de 1941 a 1986.

\begin{tabular}{|c|c|c|c|c|c|c|c|}
\hline $\begin{array}{l}\text { TRABALHOS } \\
\text { PUBLICADOS }\end{array}$ & $\begin{array}{c}1941-50 \\
n^{0}\end{array}$ & $\begin{array}{c}1951-60 \\
n ?\end{array}$ & $\begin{array}{c}1961-70 \\
n^{0}\end{array}$ & \begin{tabular}{|c|}
$1971-80$ \\
$\mathrm{n}^{0}$
\end{tabular} & \begin{tabular}{|c|}
$1981-86$ \\
$\mathrm{n}^{0}$
\end{tabular} & \begin{tabular}{|c|} 
TOTAL \\
$\mathrm{n}^{0}$
\end{tabular} & $\%$ \\
\hline Revista Brasileira de Enfermagem & 01 & 03 & 05 & 06 & 01 & 16 & 25,00 \\
\hline Revista Paulista de Enfermagem & - & - & - & - & 02 & 02 & 3,13 \\
\hline $\begin{array}{l}\text { Revista da Escola de Enfermagem } \\
\text { da Universidade de S. Paulo } \\
\text { Revista Enfermagem Novas }\end{array}$ & - & - & - & $01^{*}$ & $02^{*}$ & $03 *$ & 4,68 \\
\hline Dimensões & - & - & - & 01 & - & 01 & 1,57 \\
\hline $\begin{array}{l}\text { Revista Gaúcha de Enfermagem } \\
\text { Revista Brasileira de Saúde }\end{array}$ & - & - & - & 02 & 01 & 03 & 4,68 \\
\hline $\begin{array}{l}\text { Ocupacional } \\
\text { SOS Saúde Ocupacional e }\end{array}$ & - & - & - & - & $03^{*}$ & 03 & 4,68 \\
\hline Segurança & - & - & - & - & - & - & - \\
\hline $\begin{array}{l}\text { Fundacentro Atualidades em Pre- } \\
\text { venção de Acidentes - BI. }\end{array}$ & - & - & - & - & 02 & 02 & 3,13 \\
\hline $\begin{array}{l}\text { Catálogo do CEPEN } \\
\text { Anais de Congresso de }\end{array}$ & - & - & - & $02^{*}$ & $06^{*}$ & 08 & 12,50 \\
\hline $\begin{array}{l}\text { Enfermagem } \\
\text { Programas de Congressos de }\end{array}$ & - & - & - & - & $01 *$ & 01 & 1,57 \\
\hline Enfermagem & - & - & - & $01^{*}$ & $15^{*}$ & 16 & 25,00 \\
\hline Anais do CONPAT & - & - & - & 09 & - & 09 & 14,6 \\
\hline TOTAL & 01 & 03 & 05 & 22 & 33 & 64 & 100,00 \\
\hline
\end{tabular}

· (*) Também publicado em outra Revista 
Análise dos dados, por amostragem, demonstra-nos que no período de 1941 a 1986 foram divulgados, no país, sessenta e quatro (64) trabalhos sobre Enfermagem Ocupacional.

Selecionamos, como fonte de pesquisa, as Revistas de maior divulgação entre a classe de Enfermagem, a saber:

a. Revista Brasileira de Enfermagem - RBEn;

b. Revista Paulista de Enfermagem;

c. Revista da Escola de Enfermagem da Universidade de São Paulo - REUSP;

d. Revista Enfermagem Novas Dimensões;

e. Revista Gaúcha de Enfermagem;

f. SOS - Saúde Ocupacional e Segurança;

g. Revista Brasileira de Saúde Ocupacional.

Além destas destacamos o Catálogo do Centro de Pesquisas em Enfermagem, Programas e Anais de Congresso Brasileiros de Enfermagem, Anais do Congresso Nacional de Prevenção de Acidente do Trabalho e Boletim da Fundacentro - Atividades em Prevenção de Acidentes.

No total foram levantados 64 (sessenta e quatro) publicações, entre fascículos e periódicos e publicações seriadas.

Do resultado da análise dos dados, verificamos que houve um predomínio de publicações de trabalhos sobre a Enfermagem Ocupacional no período de 1971 a 1986, o que é plenamente compreensível diante de fatores que podem ser apontados como responsáveis pelo fato. Dentre eles, destacamos os dispositivos legais instituídos pelo Governo Federal, mantendo a obrigatoriedade de Serviços Especializados em Segurança, Higiene e Medicina do Trabalho (SESMT) nas empresas. Referindo-nos à Portaria $3237 / 72$ que define as características do pessoal especializado, quanto às atribuições e qualificações; Portaria 3460/75.

A Lei 6514/77 que dá nova redação ao capítulo $\mathrm{V}$ do título 11 da CLT em relação à Segurança e Medicina do Trabalho, além da Portaria 3214/78 que aprova as Normas Regulamentadoras do capítulo II, relativas à Segurança e Medicina do Trabalho.

Nos casos em que apareceram trabalhos publicados em mais de uma publicação, considerou-se, para registro, $o$ artigo que foi editado em primeiro lugar por ordem cronológica. No quadro ilustrativo esses foram caracterizados apenas por um asterisco.

A Revista Brasileira de Enfermagem (RBEn) órgão de divulgação da Associação Brasileira de Enfermagem (ABEn), foi criada em 1932, com a denominação de Anais de Enfermagem. Tem, atualmente, uma periodicidade trimestral e é indexada no Internacional Nursing Index. Conforme observa-se na tabela 1, a referida revista divulgou no período de 1946 a 1986, dezesseis (16) artigos sobre Enfermagem Ocupacional, com um número significativo de dezesseis (16) artigos no período de 1971 a 1980 , o que representa $25 \%$ do total publicado em 40 anos, pela Revista.
A Revista Paulista de Enfermagem, órgão oficial da ABEn - São Paulo, criada em 1980, foi indexada em 1981 no International Nursing Index. observa-se que no período de 1980 a 1986 publicou apenas dois (02) artigos sobre Enfermagem Ocupacional, correspondendo a $3,13 \%$ das publicações sobre Enfermagem Ocupacional, no período de 1941 a 1986 .

A Revista da Escola de Enfermagem da Universidade de São Paulo (REUSP), com sua publicação iniciada em 1967 é também indexada no Internacional Nursing Index.

Verificou-se que a referida Revista publicou (03) três artigos sobre Enfermagem Ocupacional, o que significa 4,68 do total da produção científica levantada.

A Revista Gaúcha de Enfermagem, órgão da Escola de Enfermagem da Universidade Federal do Rio Grande do Sul, tem data inicial em 1976 e é de periodicidade semestral. Desde o início de sua publicação divulgou três (03) artigos sobre Enfermagem Ocupacional, o que corresponde a $(4,68 \%)$.

A Revista de Enfermagem Novas Dimensões, órgão da Universidade de São Paulo, iniciou sua publicação em 1975. Deixou de circular em 1979. Contribui com apenas um (01) artigo sobre Enfermagem Ocupacional, o que corresponde a $1,57 \%$ da produção científica.

A Revista Brasileira de Saúde Ocupacional e a Fundacentro - Atualidades em Prevenção de Acidentes são órgãos de divulgação da Fundacentro - Fundação .Jorge Duprat Figueiredo - de Segurança e medicina do Trabalho - FUNDACENTRO - instituída em 1966, com a sua publicação inicial em 1973. Indexada no C.I.S. Abstract. A Revista Brasileira de Saúde Ocupacional contribui com (03) três artigos $(4,68 \%)$ e o Atualidades em Prevenção de Acidentes colaborou com 02 artigos $(4,68)$ sobre Enfermagem Ocupacional.

A Revista SOS - Saúde Ocupacional e Segurança, órgão da Associação Brasileira para Prevenção de Acidentes (ABPA) - criada em 1941 - teve sua publicação inicial em 1966. Não encontramos qualquer artigo publicado sobre Enfermagem Ocupacional, nessa conceituada revista.

Os Catálogos de Enfermagem publicados pelo Centro de Pesquisas em Enfermagem (CEPEN), relaciona teses e dissertações de pós-graduação ou de concursos para prof essor titular e teses de livre docência. Relata todas as informações sobre o trabalho, como: resumo, objetivo, metodologia e resultados. Esclarece local de defesa do trabalho e onde conseguir cópia do mesmo. Constitui-se em importante fonte de consulta da comunidade científica da Enfermagem. Seu primeiro volume foi editado em 1979. Possui, atualmente, cinco (05) volumes com 337 referências. "Destas, 1 (0,3\%) é de licenciado, 263 (78\%) são dissertações de Mestrado, $36(10,7 \%)$ são teses de Doutorado, $33(09,8 \%)$ teses de livre docência de 4 (1,2\%) são teses de Professor catedrático e titular".

Das 337 referências, foram encontradas apenas 08 
(12,50\%) disserções sobre saúde ocupacional.

Os Anais dos Congressos Nacionais de Prevenção de Acidentes - CONPAT publicados à partir de 1962. Deixou de circular em 1982. Contribuiu com 09 (nove) artigos (14,06\%).

Os Anais dos Congressos Brasileiros de Enfermagem, circulam ininterruptamente desde 1977. Constituem-se em importante fonte de informações, uma vez que divulgam os trabalhos ou resumos referentes à temática central do evento, representando a preocupação dos profissionais da categoria, naquele determinado momento. Sua triagem é restrita ao número de sócios da ABEn. Nesses Anais, localizamos 01 $(1,57 \%)$ referências sobre Enfermagem Ocupacional.

Por outro lado, os programas dos Congressos divulgam a temática central do encontro. Neles, no período de 1981 a 1986 foram relacionados 16 (dezesseis) trabalhos $(25,00 \%)$.

\section{CONCLUSÕES}

Considerando as questões propostas e os resultados obtidos, concluímos que:

Os Enfermeiros estão elaborando e divulgando trabalhos científicos na área da Enfermagem Ocupacional, embora o volume de sua produção ainda seja bastante reduzido. Já existe certa preocupação por parte de estudiosos dessa especialidade de reconhecida importãncia;

Constatamos que a elaboração teórica da enfermagem sofre a influência da pobreza bibliográfica, face ao quantitativo de material publicado;

A concentração bibliográfica significativa deu-se no período de 1971 a 1986 ;

A não divulgação de pesquisas realizadas interfere na continuidade de estudos nessa especialidade;

O desconhecimento de publicações, dificulta a busca de localização das mesmas, para enriquecimento e fundamentação de trabalhos, prejudicando com isso os estudiosos que buscam apoio nas pesquisas já realizadas.

\section{REFERÊNCIAS BIBLIOGRÁFICAS}

1. AGUIARI, C. S. A. L. Periódico para o Curso de Graduação em Enfermagem e obstetrícia no Brasil. Estudo bibliométrico. 1966-76. Revista Brasileira de Enfermagem. Brasília, 34: 333-42, 1981.

2. ALMEIDA, M.H. O conhecimento dos enfermeiros sobre os resultados de pesquisa em enf ermagem. Simpósio. In: $S E M I$ NÁRIO NACIONAL DE PESQUISA EM ENFERMAGEM. Anais... São Paulo, 1985. p. 243-59.

3. ANGERAMI, E. L. \& ALMEIDA, M. C. P. Divulgação do conhecimento científico produzido na Enfermagem. In: $S E M I$ NÁRIO SOBRE ENSINO DE PÓS-GRADUAÇĀO E PESQUI-
SA EM ENFERMAGEM.. Avaliação e perspectivas. Brasília, 1982. Relatório. p. 108-27.

4. ASSOCIAÇÃO BRASILEIA DE ENFERMAGEM. Centro de Estudos e Pesquisas em Enfermagem. Informaçies sobre Pesquisas e Pesquisadores em Enfermagem. Ribeiro Preto, 1979-1985. $5 \mathrm{v}$.

5. BRASIL. SEPLAN. CNPq. Avaliação e Pespectivas 1978. Sub Área de Enfermagem. Brasília, 1978.

6. CASTRO, I. B et alii. Dificuldades na Incorporação dos Resultados de Pesquisa na Prática de Enfermagem. Simpósio. In: SEMINÁRIO NACIONAL DE PESQUISA EM ENFERMAGEM. São Paulo, Anais... São Paulo, 1985. p. 193-242.

7. CARVAlHO, M. B. P. \& BARRET(), A. A. Publicações Primárias: alguns indicadores estatísticos da comunicação científica e tecnológica no Brasil. in: CONGRESSO LATINO AMERICANO DE BIBLIOTECNOMIA, 1., Salvador. 1980. Anais.. Salvador, MEC/CAPES, 1980. p. 559-79.

8. CIANCIARULlO, T. I. Prioridade e Limitações da Pesquisa em Enf ermagem no País. In: SEMIMÁRIO NACIONAL DE PES QUISA EM ENFERMAGEM. Ribeirão Preto. 1979. Relatório. Ribeirão Preto, 1979. p. 107-15.

9. FERREIRA. D. V. Transferência de informação. in: CONGRESSO LATINO AMERICANO DE BIBLIOTECONOMIA, 1. Salvador. 1980. Anais... Salvador, 1980.

10. _ Caracterização de uma Comunidade Científica através da literatura publicada: Um estudo de caso. Ciência da Informação, Brasília, 10 (02): 43-64, 1982.

11. FERREIRO, L. \& MENDÉZ, A. La produtividade de los autores del CSIC durante el año 1977: Estudo Sociométrico. Rev. Esp. Doc. Cient.,31: 10-24, 1980.

12. FURTADO, S. J. Conceitos e Tipologia em Ciência e Tecnologia e sua Influência na Publicação de Informaçōes. Ciência da Informação, Brasília, 10 (1): 13-8, 1981.

13. LANCASTER, F. W. Acessibilidade de Informações na Pesquisa científica em processo. Ciência da Informação, Rio de Janeiro, 4 (2): 109-17, 1975.

14. MOREL, R. L. M. \& MOREL, C.M. Um estudo sobre a produção científica brasileira segundo os dados do Institute for Scientific Information (ISI). Ciência da Informação, Rio de Janeiro, $6(2)$ : 99-109, 1977.

15. NÓBREGA, M. R. S. Mecanismos facilitadores para a utilização da pesquisa na prática de enfermagem e para divulgação dos resultados das pesquisas realizadas. Simpósio. In: SEMINÁRIO NACIONAL DE PESQUISAS EM ENFERMAGEM. São Paulo. Anais... São Paulo, 1985. p. 260-75.

16. PAIM, L. Prioridades e limitações da pesquisa em Enfermagem no país. Painel. In: SEMINÁRIO NACIONAL DE PESQUISA EM ENFERMAGEM. Ribeirão Preto. 1979, Relatório. Ribeirão Preto, 1979. p. 119-33.

17. RODRIGUES, A. P. S. Possibilidades e limitações da pesquisa em Enf ermagem no Brasil: Estudo comparativo entre mestras e mestrandas sobre suas dissertaçōes de mestrado. Rio de Janeiro, Universidade Federal do Rio de Janeiro, 1981. $214 \mathrm{p}$.

18. SANTOS, M. C. P. Características dos pesquisadores brasileiros de química e a sua produção científica no periodo de 1973 a 1977. Rio de Janeiro, Instituto Brasileiro de Ciência de Tecnologia, Universidade Federal do Rio de Janeiro, 1981. 1399 p.

19. WRIGHT, M.G. et alii. Avaliação e perspectivas. Área de Saúde. Sub-área Enfermagem. Relatório. Brasília, CNPq/CAPES, 1981

AMOSTRAGEM DA LITERATURA CIENTIFICA SOBRE ENFERMAGEM OCUPACIONAL, NO PERIODO DE 1941-1986.

\section{ARTIGOS DE PERIÓDICOS}

1946

DOURADO, H. Enfermagem Industrial. Revista Brasileira de Enfermagem, São Paulo, 15 (20): 16-19, jun/set. 1946. 
1958

CASTR(), I. B. Enfermagem em higiene industrial. Revista Brasileira de Enfermagem, Rio de Janeiro, 11 (:3): 221-233, set. 1958 .

SPERA, F. C. Enfermagem Industrial. Formação e treinamento de enfermeira. Revista Brasileira de Enfermagem, Rio de Janeiro, 11 (1): 52-59, mar. 1958.

1960

CORDEIR(), I. s. et alii. () Serviço de enfermagem da Divisão de Saúde da Indústria e Comércio de Minérios S/A - IC(OMI. Revista Brasileira de Enfermagem, Rio de Janeiro, $1: 3$ (3): :362-381, set. 1960).

1961

()()RIDEIR(), D. S. \& FERREIRA, M. L. S. A enfermagem em Saúde Pública em uma comunidade industrial rural. Revista Brasileira de Enfermagem, Rio de Janeiro, 14 (4) 368-373, ago. 1961 .

1963

ARRUI)A, M. J. B. Enfermagem Industrial. Revista Brasileira de Enfermagem, Rio de Janeiro, 16 (:3): 1:31)-1:3:3, jun. 196:3.

\section{6}

ALVIM, E. F. A Enfermagem no Serviço de Saúde ()cupacional. Revista Brasileira de Enfermagem. Rio de Janeiro, 2: $267-267,1986$

CORIEIR(), I. S. Visita ao operário no local de trabalho (V()LT) Revista Brasileira de Enfermagem. Rio de Janeiro, 1.9 (2/3): 114-12:3, abr.jun. 1966.

1967

MAGALHÃES, M. M. \& ALVIM, E. F. Saúde ()cupacional: um campo aberto ao trabalho do enfermeiro. Revista Brasileira de Enfermagem, 20 (4): :37:3-38:2, ago. 1967.

1975

I)ANTAS, I. C. Estudo e Análise do Programa Atual do Curso de Especialização para Enfermeiro do Trabalho, In: $C(O N$ GRESS() NACI()NAL DE PREVENÇÃ) DE ACIDENTES D() TRABALH(), 14, Rio de Janeiro, 1975. Anais... São Paulo, Arte e texto, 1976. p. 149-(6).

PEG(I)RAN(), A. S. Curso para Auxiliares de Enfermagem do Trabalho. In: C(ON(GRESS() NACIONAL DE PREVENÇAO() DE ACIDENTES DE TRABALHO, 14, Rio de Janeiro, 1975. Anais... São Paulo, Arte e Texto, 1976. p. 167-174.

SCHMIDT, M. J. Subsídios para instalação de um serviço para acidentes do trabalho. Revista Brasileira de Enfermagem, Rio de .Janeiro, 28 (1): 65-71, jan./mar.. 1975.

1976

ANIRADE, I. K. (). I). et alii. A enfermagem do trabalho em serviços de Medicina ()cupacional. In: CONGRESS() NACI()NAL DE PREVENÇÃO DE ACIDENTES D() TRABALHO, 15, Belo Horizonte, 1976. Anais... São Paulo, Novos Horizontes, 1977, p. 729-38.

I)()URADO, H. G. A Enfermagem na Conferência Internacional do Trabalho. Revista Brasileira de Enfermagem, Brasília 29 (2): 5, abr.jun. 1976.

MAUR(), M. Y. C. et alii. Fadiga e aspectos ergônomicos do trabaIho' de enfermagem. Revista Brasileira de Enfermagem, Brasília, 29 (4): 7-28, out./dez. 1976.

SATO, A. et alii. Contribuição para o estudo das funções e atribuições do Enfermeiro do Trabalho. In: CONGRESSO NACIONAL DE PREVENÇÃO DE ACIDENTES D() TRABALHO, 15, Belo Horizonte, 1976. Anais... São Paulo, Novos Horizontes, 1977 , p. $729-38$.

(OGUISSO, T. \& SCHMIDT, M. J. A propósito da Classificação Brasileira Uniforme de Ocupações. Revista Brasileira de En-

(*) Também divulgada em outra publicação. fermagem, Brasília, 2.9 (2): 6-23. abr./jun. 1976.

S()ARES, C. B. \& LIMA, I. S. Enfermagem na higiene do trabalho. Revista Gauicha de Enfermagem, Porto Alegre. 1(3): 207-30, 1976 .

\section{7}

ANIRADE, I. K. (). I. et alii. Um Manual de Procedimentos de Enfermagem do Trabalho. In: CONGRESS() NACIONAL DE PREVENÇÃ) DE ACIDENTES DO TRABALH(), 16, Porto Alegre, 1977. Anais... São Paulo, Fundacentro, 1978, p. 191-219.

('ARVAlH(), A. C. Condições de Trabalho do pessoas de Enfermagem 6 la. conf erência da ()rganização Internacional do Trabalho (OIT) - Comunicação. Revista Brasileira de Enfermagem, Brasília 30(2): 157-161, abr./jun. 1977.

MENELLI, E. R. \& KAESTNER, M. Enfermagem do trabalho. Revista Gaúcha de Enfermagem, Porto Alegre, 2 (1): 17-35. 1977.

1978

ANDRADE, A. S. R. et alii. Atribuições da (o) enfermeira (o) do trabalho. Revista de Enfermagem Novas Dimensões, São Paulo, 4 (6): 331-333, 1978.

FUZISK(), A. K. et alii. Imunização Antitetãnicaa em Serviços Médicos da Empresa, In: C()NGRESS() NACI()NAL DE PREVENÇÄ() DE ACIDENTES D() TRABALH(), 17 São Paulo, 1978. Anais... São Paulo, FUNDACENTRO, 1979 p. 389-392.

VENDRÚSCUL(), E. M. A importãncia da observação como "arma" da enfermeira do trabalho no desempenho de suas funçōes. Revista Brasileira de Enfermagem, Brasília, 31: $7-12,1978$.

1979

FI(iUEIREI)(), A. Askaréis, Riscos, controles, manuseio e destinação final. In: C(NGRESS() NACIONAL DE PREVENÇÃ) DE ACIDENTES DO TRABALHO, 18 Salvador, 1979. Anais... São Paulo, FUNDACENTRO, 1980 p. 433-440.

FRIEILANIER, V. M. \& SIMMELINK. L. M. Aplic'ação da Função Assistencial de Enfermagem do Trabalho pelos Serviços de Enfermagem do Trabalho. In: CONGRESSO NACIONAL DE PREVENÇÃO DE ACIDENTES DO TRABALHO, 18, Salvador. 1979. Anais... São Paulo, FUNDACENTRO, 1980 p. 333-340.

MAURO, M. Y. C. Fadiga e o Trabalho Docente de Enfermagem. In: CONGRESSO BRASILEIRO DE ENFERMAGEM, 31, Fortaleza, 1979. Fortaleza, ABEn, 1979. p. 58-59.

SIMMELINK, L. J. M. Sistemática de Implantação de Serviços de Enf ermagem do trabalho. In: CONGRESSO NACIONAL DE PREVENÇÃO DE ACIDENTES DO TRABALHO, 18, Salvador, 1979. Anais... São Paulo, FUNDACENTRO, 1980 p. 590.

\section{0}

QUEIROZ, W.M Organização de Serviços de Enfermagem do Trabalho. Revista da Escola de Enfermagem da Universidade de São Paulo, São Paulo, 14 (2): 197. 1980

\section{1}

QUEIROZ, W.M. Acidentes do Trabalho nos Hospitais. Revista Paulista de Enfermagem, São Paulo, 1 (1): 23-25, 1981.

1982

NEVES, T.A. et alii. 0 papel do(a) enf ermeiro (a) do Trabalho na reniserção social da pessoa com deficiência, Revista Brasileira de Enfermagem, Brasília, 35 (3): 192-199, jul./dez. 1982.

NOGUEIRA, M.J.C. Níveis de Prevenção em Enfermagem do Trabalho. Revista da Escola de Enf ermagem da USP, São Paulo, 16 (3): 275-84, 1982. 
1983

NOGUEIRA, M. J. C. Níveis de Prevenção em Enfermagem do Trabalho. Revista Brasileira de Saúde Ocupacional, São Pau. lo, 48 (11): 57-61. 1983 (ver item 32).

SILVA, M.P. Absenteísmo: grande problema das chefias de enf ermagem. Revista Gaúcha de Enfermagem, Porto Alegre, 1 (4): 85-98, 1985.

\section{4}

ALVEZ, C. Objetivos e atividades do Serviço de Enfermagem Ocupacional em Empresa Siderúrgica. FUNDACENTRO. Atualidades em Prevenção de Acidentes, 15 (173) maio, 1984.

FIGUEIREDO, N. M. A. Fadiga, Intercorrência na Assistência da Enfermagem. In: CONGRESSO BRASILEIRO DE ENFERMAGEM 36, Belo Horizonte, 1984. ABEn, Belo Horizonte, 1984 , p. 65.

LEIFERT, R.M.C. \& FRIEDLANDER, V. Relações do Trabalho e Saúde do Trabalhador In: CONGRESSO BRASILEIRO DE ENFERMAGEM, 36, Belo Horizonte, 1984, Anais... Belo Horizonte, ABEn, 1984, p. 47-52. *

OGUISSO, T. \& SCHMIDT, M. J. A Enfermagem e a insalubridade. Revista Paulista de Enfermagem, São Paulo, 4 (2): 43-48, 1984

SOUSA. M. S. B. Enf ermagem do Trabalho: Um estudo exploratório em empresas na cidade de Belém. In: CONGRESSO BRASILEIRO DE ENFERMAGEM, 36, Belo Horizonte, 1984 ABEn. Belo Horizonte, 1984. p. 41.

LEIFERT, R. M. C. \& FRIEDLANDER, V. M. Relações de Trabalho e a Saúde do Trabalhador. Revista Brasileira de Saúde Ocupacional, 12 (48): 58-60. 1984. (ver item $36^{*}$ ).

1985

ARAÚJO, E. A. G. et alii. Estudo para revisão da programação do Curso de Auxiliar de Enfermagem do Trabalho. In: $\mathrm{CON}$ GRESSO BRASILEIRO DE ENFERMAGEM. 1985. p. 37 Recife, ABEn, 1985, n. 59.

LEIFERT, R. M. C. et alii. Hospital e Insalubridade, Riscos de Insalubridade para o pessoal médico e paramédico. In: $\mathrm{CON}$ GRESSO BRASILEIRO DE ENFERMAGEM, 37, Olinda 1985, Recife, ABEn, n. 137, 1985.

PROGRAMAÇÃO DE ENFERMAGEM DO TRABALHO Recomendação do II Encontro Paulista de Enfermeiros do Trabalho. FUNDACENTRO Atualidades em Prevenção de Acidentes 16 (191) nov. 1985.

ROBAZZI, M. L. C. C. et alii. Considerações sobre o Serviço dos Coletores de lixo de uma Cidade do Interior do Estado de São Paulo. In: CONGRESSO BRASILEIRO DE ENFERMAGEM, 37, Olinda, ABEn, 1985. № 174. *

Coletores de Lixo: estudo de afastamento do serviço por problemas de saúde. Revista Brasil€ira de Saúde Ocupacional 13 (50): 68-74. 1985. (ver item 42)*.

SOUSA, M. S. B. Enf ermagem Ocupacional: Uma necessidade na formação do enf ermeiro. In: CONGRESSO BRASILEIRO DE ENFERMAGEM, 37, Olinda, ABEn, 1981, n? 171.

SILVA, V. E. F. C'ausas do Afastamento do Serviço por Acidentes do Trabalho. Revista da Escola de Enf ermagem da USP. São Paulo 19 (2): 187-188. 1985. *

\section{6}

BAPTISTA, S. de S \& CASTRO, J.B. O estudante de Enfermagem e a Saúde do Trabalhador. In: CONGRESSO BRASILEIRO DE ENFERMAGEM, 38 Rio de Janeiro, 1986. ABEn, Rio de Janeiro, n. 099

(*) Também divulgada em outra publicação.
BARBOSA, A. L. et. alii. Atuação da equipe de Saúde Ocupacional do CENPES diante de Acidentes Oculares Químicos em laboratórios. In: CONGRESSO BRASILEIRO DE ENFERMAGEM, 38, Rio de Janeiro, ABEn n. 155.

MACIEL, A. L. et alii. Projeto de Implantação de Assistência de Enfermagem em Saúde Ocupacional. In: CONGRESSO BRASILEIRO DE ENFERMAGEM, 38, Rio de Janeiro, 1986. ABEn Rio de Janeiro n. 262.

MONTEIRO, M. S. et. alii. Acidentes de Trabalho entre o pessoal de Enfermagem de um hospital universitário. In: $\mathrm{CON}$ GRESSO BRASILEIRO DE ENFERMAGEM, 38, Rio de Janeiro, 1986. ABEn Rio de Janeiro. n. 151.

PAULUCCI, M. H. et. alii. Saúde Ocupacional e Enfermagem: Algumas consideraçōes. In: CONGRESSO BRA SILEIRO DE ENFERMAGEM, 38, Rio de Janeiro, ABEn, Rio de Janeiro, n. 190.

SILVA, A. \& BIANCHI, R. F. Estresse Ocupacional do Enfermeiro de Centro de material. In: CONGRESSO BRASILEIRO DE ENFERMAGEM, 38, Rio de Janeiro, 1986, ABEn. Rio de Janeiro. n. 125.

SOARES, E. \& PEIXOTO, J. C. Graus de risco a que estão expostos os trabalhadores em instituições hospitalares - Modelo de intervenção de enfermagem centrado nos riscos dominantes. In: CONGRESSO BRASILEIRO DE ENFERMAGEM, 38, Rio de Janeiro, ABEn, Rio de Janeiro. n. 150.

SOUSA, M. S. B. Vicessitudes da Enfermagem do Trabalho: In: CONGRESSO BRASILEIRO DE ENFERMAGEM, 38, Rio de Janeiro, 1986, ABEn, Rio de Janeiro. n. 152.

VIGNALI, E. M. Enfermagem do Trabalho. Reflexão sobre aspectos: filosóficos, históricos e de saúde mental. In: CONGRES SO BRASILEIRO DE ENFERMAGEM. 38, Rio de Janeiro, ABEn, Rio de Janeiro n. 265

\section{DISSERTAÇŌES}

1977

MAURO, M. Y. C. Fadiga e o trabalho docente da Enfermagem. Rio de Janeiro, UFRJ. 1977, 159 p. Tese para obtenção do título de livre docente, (ver item 27)*

1979

QUEIROZ, W. M. Organização de Serviços de Enfermagem do Trabalho. São Paulo. Faculdade de Saúde Pública da USP. 1979. p. 112. Diss. de mestr. (ver item 29) *.

1980

POSSO, M. B. S. O ruído emitido por aparelhos usados nas salas de operação. São Paulo, Escola de Enfermagem da USP, 1980. 100 p. Diss. mestr.

SOUSA, M. S. B. Enf ermagem do Trabalho: Um estudo exploratório em empresas do município de Belém. Rio de Janeiro - UFRJ, 1980. 109 p. Diss. de mestr. (ver item 38) *.

1982

TESCK, E. C. B. Convivência contínua com estresse - Vida e trabalho de enfermeiros na UTI. Rio de Janeiro, UFRJ, 1982. 110 p. Diss. de mestr.

1984

ROBAZZI, M. L. C. C. Estudo das Condições de vida, trabalho e risco sujeitos os coletores de lixo da cidade de Ribeirão Preto. São Paulo, USP. 1984. 132 p. Diss. mestr. (ver item 42)*

SIQUEIRA, M. D. C. Fatores predisponentes ao obsenteísmo em Enf ermagem, Rio de Janeiro, UFRJ, 1984. 98 p. Diss. mestr.

1986

VIGNALI, E. M. Enfermagem do Trabalho: Reflexão sobre aspectos filosóficos, históricos e de saúde mental. Ribeirão Preto, USP, 1986. Diss. de mestr. (ver item 53) * . 